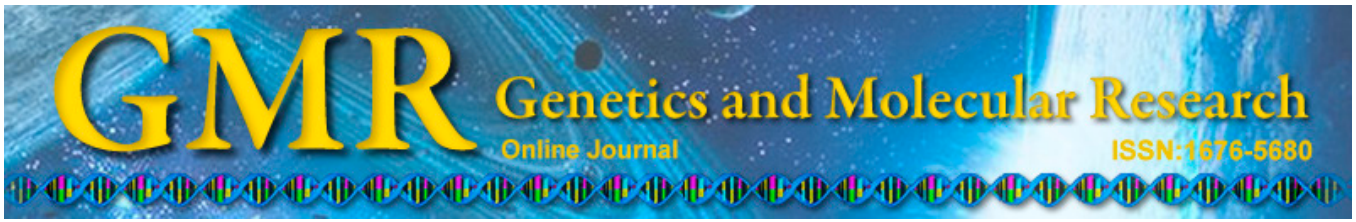

\title{
Novel NPHS1 splice site mutations in a Chinese child with congenital nephrotic syndrome
}

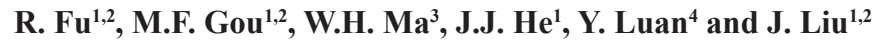 \\ ${ }^{1}$ Department of Pediatrics, Puyang Youtian General Hospital, \\ Puyang Henan, China \\ ${ }^{2}$ Department of Pediatrics, Affiliated Puyang Youtian General Hospital, \\ Xinxiang Medical University, Puyang Henan, China \\ ${ }^{3}$ Department of Clinical Laboratory, Puyang Youtian General Hospital, \\ Puyang Henan, China \\ ${ }^{4}$ College of Laboratory Medicine, Dalian Medical University, Dalian, \\ Liaoning, China \\ Corresponding author: R. Fu \\ E-mail: fuurong2005@163.com
}

Genet. Mol. Res. 14 (1): 433-439 (2015)

Received January 22, 2014

Accepted April 14, 2014

Published January 23, 2015

DOI http://dx.doi.org/10.4238/2015.January.23.17

\begin{abstract}
Congenital nephrotic syndrome (CNS) is defined as heavy proteinuria or nephrotic syndrome occurring before 3 months of age. It is characterized by early onset and progresses to end-stage renal disease. Recently, several genes associated with CNS have been identified, including NPHS1 and NPHS2. Mutations in the NPHS1 gene have been identified in patients with CNS in Finland with relatively high frequency. Thus far, only a few case reports about CNS have described an NPHS1 mutation in China. In this study, mutational analyses of NPHS1 and NPHS2 were performed in a Chinese child with CNS. Mutations were analyzed in all exons and exon/intron boundaries of NPHS1 and NPHS2 in the patient and his parents as well as in 50 unrelated controls using polymerase chain reaction and
\end{abstract}


direct sequencing techniques. No mutations were detected in NPHS2. A novel splice site mutation (IVS11+1G $>$ A) within intron 11 and a missense mutation within exon 8 (c.928G $>$ A) in the NPHS1 gene were detected in the child. The child's mother had normal urinalysis and a c. $928 \mathrm{G}>\mathrm{A}(\mathrm{D} 310 \mathrm{~N})$ heterozygous mutation, and his father had normal urinalysis and IVS11+1G $>$ A. These were not identified in the 50 unrelated controls. The novel splice site mutation of IVS11+1G $>$ A and a missense mutation at c. $928 \mathrm{G}>\mathrm{A}$ in $N P H S 1$ were found to cause CNS in this Chinese child.

Key words: Chinese; Congenital nephrotic syndrome; NPHS1

\section{INTRODUCTION}

NPHS1 is localized on chromosome 19q13.1 and codes for the nephrin protein, an essential component of the interpodocyte-spanning slit diaphragm (SD) (Kestilä et al., 1998). Nephrin is a transmembrane protein of the immunoglobulin (Ig) superfamily; it contains an extracellular domain with 8 IgG-like motifs and a fibronectin type-like motif, a transmembrane domain, and a cytosolic C-terminal end (Kestilä et al., 1998). Nephrin forms a zipperlike filter structure in the center of the slit and plays an important role in cell-cell signaling in the SD (Pätäri-Sampo et al., 2006).

The NPHS1 gene has been identified as the causative gene of congenital nephrotic syndrome (CNS) of the Finnish type (CNF), a rare autosomal recessive disorder. This disease is characterized by massive proteinuria, a large placenta, and the onset of nephrotic syndrome within the first 3 months of the life. The course of CNF is progressive, often leading to endstage renal disease within 2-3 years of age (Kestilä et al., 1998; Heeringa et al., 2008).

Mutations in the NPHS1 gene lead to disruption of the filtration barrier and cause massive protein loss. To date, more than 160 different mutations, including deletions, insertions, nonsense, missense, splice site, and promoter mutations, have been reported both in Finnish and non-Finnish patients (Lenkkeri et al., 1999; Beltcheva et al., 2001; Yu et al., 2012; Ameli et al., 2013).

NPHS1 is relatively common in the Finnish population, with an incidence of 1:8200 newborns (Ahvenainen et al., 1956; Norio, 1966). This frequency is much lower in other countries (Fuchshuber et al., 1996). The mutation detection rate of NPHS1 approaches $98 \%$ in Finnish cases. However, NPHS1 genetic screening in patients of non-Finnish origin has shown a detection rate between 39-80\% (Lenkkeri et al., 1999; Hinkes et al., 2007; Heeringa et al., 2008; Ismaili et al., 2009; Schoeb et al., 2010). Only a few cases regarding NPHS1 mutations in CNS have been reported in China (Shi et al., 2005; Wu et al., 2011; Yu et al., 2012). In this study, we identified novel NPHS1 splice site mutations in a Chinese child with CNS.

\section{MATERIAL AND METHODS}

\section{Patients and data recruitment}

A Chinese boy was admitted to the Department of Pediatrics, Puyang Youtian Hospital at 70 days of age for evaluation of edema, which occurred 25 days after birth. He was a 
full-term normal delivery baby with a birth weight of $2.5 \mathrm{~kg}$. The weight of the placenta was unknown. Urinalysis showed 3+ protein and microscopic hematuria (4-10 erythrocytes per high-power field). Serum albumin was $16.9 \mathrm{~g} / \mathrm{L}$ and serum cholesterol was $5.62 \mathrm{mM}$. There was no evidence of congenital infection. Screening of blood serum and urine from the patient excluded the presence of antibodies for toxoplasma, rubella virus, cytomegalovirus, herpes simplex virus, and organic acid metabolic disorder. Renal biopsy was refused by his parents. The patient was diagnosed with CNS. Both the father and the mother were 24 years old and showed normal urinalysis results. Parental consanguinity was denied. The other members of the family had no history of renal disease.

We performed mutational analysis of NPHS1 encoding nephrin and NPHS2 encoding podocin, the most frequent monogenic causes of childhood CNS. This study was approved by the Ethics Committee of the Hospital of Puyang.

\section{Mutation analysis}

Polymerase chain reaction (PCR) amplification of genomic DNA was conducted after obtaining informed consent. Peripheral blood samples from the boy and his parents as well as from 50 controls were obtained for genetic analysis in tubes containing potassium oxalate. Genomic DNA was isolated from blood samples using an E.Z.N.A Blood DNA purification kit (OMEGA Bio-Tek, Norcross, GA, USA) following manufacturer guidelines. Mutation analysis of NPHS1 and NPHS2 was performed using PCR and direct sequencing. Primers were designed to cover the sequences of all exons and introns adjacent to each exon of NPHS1 and NPHS2, based on published primer sequences (Lenkkeri et al., 1999; Tsukaguchi et al., 2002). PCR products were directly sequenced using the ABI PRISM Big Dye kit using the same amplification primers with an ABI 377 DNA sequencer (Applied Biosystems, Foster City, CA, USA).

Sequences were analyzed using the DNAStar software. For references to NPHS1 and NPHS2, the published wild-type sequences (NM_004646 and NM_014625) were used for both nucleotide and amino acid numbering. For all detected mutations and variants, both strands were sequenced. Whenever possible, segregation was confirmed by direct sequencing of the parental samples.

Urinalysis of the 50 control subjects was normal. Novel mutations of NPHS1 were investigated in the 50 healthy controls by direct sequencing

\section{RESULTS}

No mutation in NPHS2 was detected. Two heterozygous single-base mutations (c.928G $>$ A and IVS11+1G $>$ A) in NPHS1 were identified in the child with CNS. The c.928G $>$ A mutation within exon 8 has been published previously (Shi et al., 2005) and can cause an amino acid substitution (D310N). A novel splice site mutation IVS11+1G $>$ A (c1440+1G $>$ A) within intron 11 has not been previously reported; this mutation may cause a truncated nephrin molecule lacking the intracellular and transmembrane domains, whereas this was not observed in the 50 controls. Further mutational analysis of the NPHS1 gene of the parents showed a heterozygous mutation, IVS11+1G $>\mathrm{A}$, in the father (Figure 1) and a heterozygous mutation, c. $928 \mathrm{G}>\mathrm{A}$, in his mother. 

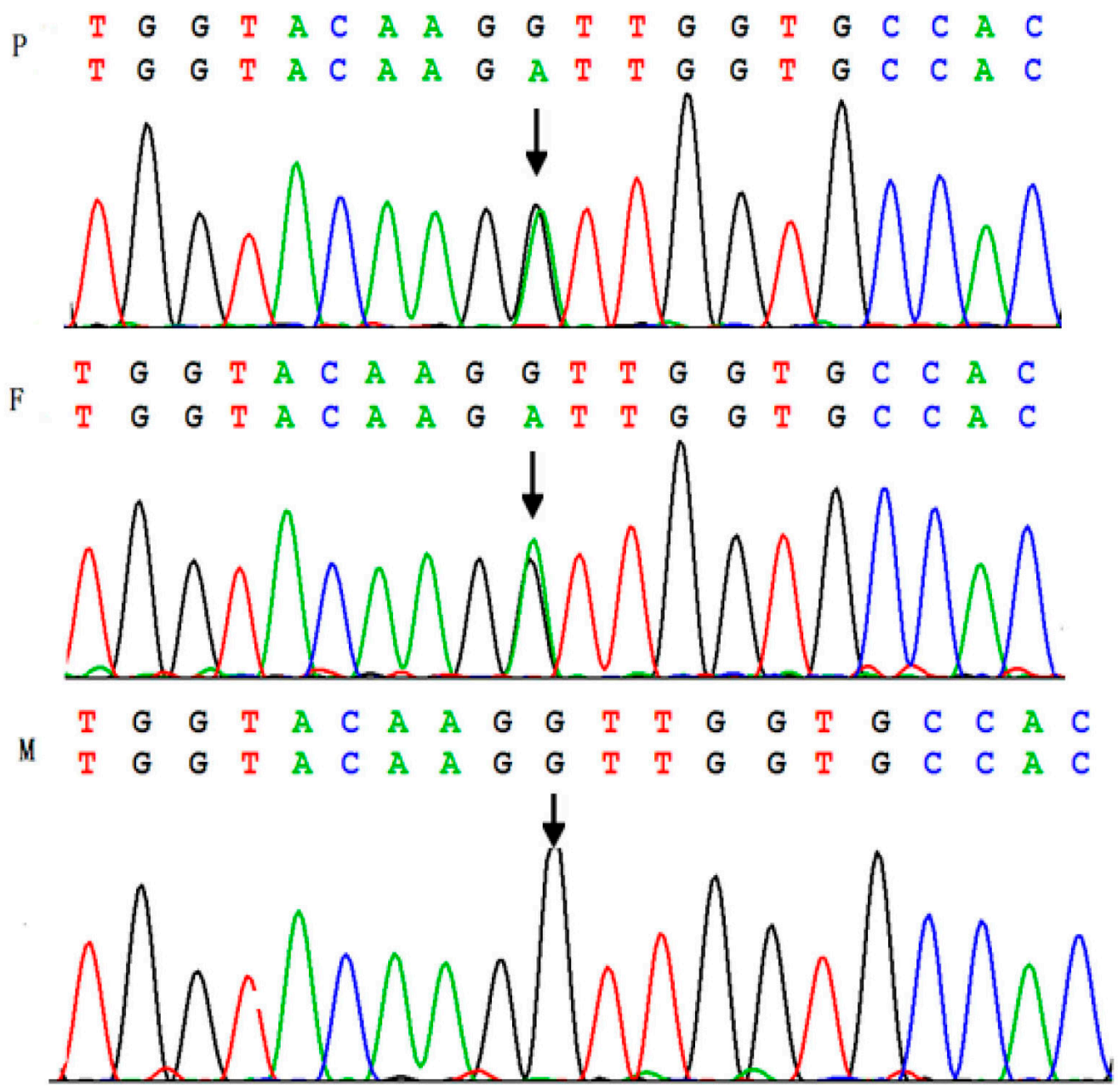

Figure 1. Novel splice site mutations IVS11+1G $>$ A of the NPHS1 gene were identified by sequencing a Chinese child with congenital nephrotic syndrome. Chromatogram by sequencing of exon 11 of NPHS1 from the patient (P), father $(\mathrm{F})$, and mother $(\mathrm{M})$. The arrows indicate mutant positions.

\section{DISCUSSION}

We found that the Chinese boy with CNS carried 2 heterozygous mutations in NPHS1, a splice-site mutation (IVS11+1G $>$ A) and a missense mutation (c.928G $>$ A). The splice site mutation (IVS11+1G $>$ A) is predicted to abrogate splice acceptor function and lead to abnormal splicing of exon 11. This mutation is located in Ig5 and may result in a truncated nephrin molecule lacking intracellular and transmembrane domains (Lenkkeri et al., 1999). This indicates that this previously undescribed mutation is indeed a pathogenic mutation. The missense mutation (c.928G $>$ A), which is predicted to result in a 310 -aspartic acid that is replaced with asparagine glutaminea in Ig3, results in misfolding and defective intracellular transport and the 
consequent absence of the mutant nephrin on the plasma membrane (Liu et al., 2001). This mutation has been previously reported and accepted as a pathological mutation (Shi et al., 2005).

NPHS2 gene mutations have also been identified as causative genes in CNS patients (Jalanko, 2009). However, we analyzed all 8 possible mutations in NPHS2, and found no causative mutations in NPHS2 in this child.

Based on our analysis, these two heterozygous mutations in the NPHS1 gene were deemed to be disease-causing mutations in this patient.

The NPHS1 gene consists of 29 exons and has a genomic size of $26 \mathrm{~kb}$, with a coding region of $4.3 \mathrm{~kb}$. The nephrin protein is composed of 1241 amino acids and is homologous to cell adhesion molecules of the Ig superfamily. Several cell adhesion molecules have been shown to have diverse functions in cell-cell or cell-matrix interactions. These molecules have an extracellular domain of 8 Ig-like modules, a fibronectin type III-like module, a single transmembrane domain, and a cytosolic C-terminal end (Kestilä et al., 1998). Nephrin plays a significant role in promoting cellular contacts through direct nephrin-nephrin interactions through the contribution of other SD components (Khoshnoodi et al., 2003). Phosphorylated nephrin binds to Nck, which is an adapter protein that is involved in reorganizing the cell's actin cytoskeleton in podocytes (Jones et al., 2006).

In 1998, mutations in NPHS1 (AF035835) were first reported to be associated with CNS by Kestilä et al. (1998). More than 160 different mutations in NPHS1, mostly in exons, have been published (Schoeb et al., 2010), of which 2 types of classical mutations are most commonly observed in Finnish patients, including Finmajor (nt121delCT, L41fsX91) in exon 2 and Finminor (c.3325C > T, R1109X) in exon 26. The NPHS1 mutation detection rate approaches $98 \%$ in children with CNS in Finland (Kestilä et al., 1998). However, outside of Finland, the NPHS1 mutation detection rate is $39-80 \%$ in cases with CNS (Lenkkeri et al., 1999; Hinkes et al., 2007; Heeringa et al., 2008; Ismaili et al., 2009; Schoeb et al., 2010). Currently, only 8 NPHS1 mutations have been described in Chinese individuals, including 1 deletion (c.1983-1900del8) (Shi et al., 2005), 1 insertion (c.3250insG) (Yu et al., 2012), 1 nonsense mutation (c.2783C $>$ A) (Wu et al., 2011), and 5 missense mutations (D310N, Q453R, I742T, R800C, V957L) (Shi et al., 2005; Mao et al., 2007; Wu et al., 2011). In this study, we found a novel splice-site mutation (IVS11+1G>A) in NPHS1 that caused CNS in a Chinese child. Information regarding the frequency and ethnic distribution of known mutations will help us to understand the pathogenesis of CNS.

We identified 2 heterozygous mutations, c. $928 \mathrm{G}>\mathrm{A}(\mathrm{D} 310 \mathrm{~N})$ in exon 8 and IVS11+1G $>\mathrm{A}$ in intron 11, of the NPHS1 gene in the Chinese patient, demonstrating that NPHS1 mutation can cause Chinese CNS. Further mutational analysis of the NPHS1 gene in the parents of the patient revealed that the 2 heterozygous mutations, c. $928 \mathrm{G}>\mathrm{A}$ and IVS11 $+1 \mathrm{G}>\mathrm{A}$, were of paternal and maternal origin. Therefore, we provided genetic counseling and prenatal diagnosis for the family, as they appeared to be at high risk.

We also detected 5 variants (c.349G $>$ A (rs3814995), c.3315G $>$ A (rs2071327), IVS $8+68 \mathrm{~A}>\mathrm{G}$, IVS24+36C $>\mathrm{T}$, and IVS $27+45 \mathrm{C}>\mathrm{T}$ ) of $N P H S 1$ in the patient, his parents, and some controls, which are thought to be NPHS1 polymorphisms. Of these, c. $349 \mathrm{G}>\mathrm{A}$, which causes a glutamic acid to lysine substitution (E117K), has been identified previously in Europeans and Chinese patients (Lenkkeri et al., 1999; Shi et al., 2005). The common polymorphism, c.3315G $>$ A, which does not result in an amino acid substitution, has previously been found in Finnish and Chinese patients (Lahdenkari et al., 2004; Shi et al., 2005). The polymorphism IVS8 $+68 \mathrm{~A}>\mathrm{G}$ has previously been found in Chinese patients (Shi et al., 2005), and 
IVS24+36C $>$ T and IVS27 $+45 \mathrm{C}>\mathrm{T}$ have been published in the SNP database of the National Center for Biotechnology in the United States.

Previously, there were only 3 articles describing cases of children with CNS that detected NPHS1 gene mutations in China. Our study expands the number of novel mutations in NPHS1. In conclusion, molecular analysis shows that NPHS1 mutations are an important cause of CNS in China.

Although CNS is uncommon in China, screening for mutations in the NPHS1 gene supports definitive diagnosis, which may be useful for clinical management of such patients as well as family counseling and family planning.

\section{ACKNOWLEDGMENTS}

We would like to thank the patient and his family for their participation in this study.

\section{REFERENCES}

Ahvenainen EK, Hallman N and Hjelt L (1956). Nephrotic syndrome in newborn and young infants. Ann. Paediatr. Fenn. 2: $227-241$.

Ameli S, Zenker M, Zare-Shahabadi A, Taher Esfahani S, et al. (2013). Novel NPHS1 gene mutation in an Iranian patient with congenital nephrotic syndrome of the Finnish type. Nefrologia 33: 747-749.

Beltcheva O, Martin P, Lenkkeri U, Tryggvason K, et al. (2001). Mutation spectrum in the nephrin gene (NPHS1) in congenital nephrotic syndrome. Hum. Mutat. 17: 368-373.

Fuchshuber A, Niaudet P, Gribouval O, Jean G, et al. (1996). Congenital nephrotic syndrome of the Finnish type: linkage to the locus in a non-Finnish population. Pediatr. Nephrol. 10: 135-138.

Heeringa SF, Vlangos CN, Chernin G, Hinkes B, et al. (2008). Thirteen novel NPHS1 mutations in a large cohort of children with congenital nephrotic syndrome. Nephrol. Dial. Transplant. 23: 3527-3533.

Hinkes BG, Mucha B, Vlangos CN, Gbadegesin R, et al. (2007). Nephrotic syndrome in the first year of life: two thirds of cases are caused by mutations in 4 genes (NPHS1, NPHS2, WT1, and LAMB2). Pediatrics 119: e907-e919.

Ismaili K, Pawtowski A, Boyer O, Wissing KM, et al. (2009). Genetic forms of nephrotic syndrome: a single-center experience in Brussels. Pediatr. Nephrol. 24: 287-294.

Jalanko H (2009). Congenital nephrotic syndrome. Pediatr. Nephrol. 24: 2121-2128.

Jones N, Blasutig IM, Eremina V, Ruston JM, et al. (2006). Nck adaptor proteins link nephrin to the actin cytoskeleton of kidney podocytes. Nature 440: 818-823.

Kestilä M, Lenkkeri U, Mannikko M, Lamerdin J, et al. (1998). Positionally cloned gene for a novel glomerular protein nephrin - is mutated in congenital nephrotic syndrome. Mol. Cell 1: 575-582.

Khoshnoodi J, Sigmundsson K, Ofverstedt LG, Skoglund U, et al. (2003). Nephrin promotes cell-cell adhesion through homophilic interactions. Am. J. Pathol. 163: 2337-2346.

Lahdenkari AT, Kestila M, Holmberg C, Koskimies O, et al. (2004). Nephrin gene (NPHS1) in patients with minimal change nephrotic syndrome (MCNS). Kidney Int. 65: 1856-1863.

Lenkkeri U, Mannikko M, McCready P, Lamerdin J, et al. (1999). Structure of the gene for congenital nephrotic syndrome of the Finnish type (NPHS1) and characterization of mutations. Am. J. Hum. Genet. 64: 51-61.

Liu L, Doné SC, Khoshnoodi J, Bertorello A, et al. (2001). Defective nephrin trafficking caused by missense mutations in the NPHS1 gene: insight into the mechanisms of congenital nephrotic syndrome. Hum. Mol. Genet. 10: 2637-2644.

Mao J, Zhang Y, Du L, Dai Y, et al. (2007). NPHS1 and NPHS2 gene mutations in Chinese children with sporadic nephrotic syndrome. Pediatr. Res. 61: 117-122.

Norio R (1966). Heredity in the congenital nephrotic syndrome. A genetic study of 57 Finnish families with a review of reported cases. Ann. Paediatr. Fenn. 12: 1-94.

Pätäri-Sampo A, Ihalmo P and Holthofer H (2006). Molecular basis of the glomerular filtration: nephrin and the emerging protein complex at the podocyte slit diaphragm. Ann. Med. 38: 483-492.

Schoeb DS, Chernin G, Heeringa SF, Matejas V, et al. (2010). Nineteen novel NPHS1 mutations in a worldwide cohort of patients with congenital nephrotic syndrome (CNS). Nephrol. Dial. Transplant. 25: 2970-2976.

Shi Y, Ding J, Liu JC, Wang H, et al. (2005). NPHS1 mutations in a Chinese family with congenital nephrotic syndrome. Zhonghua Er Ke Za Zhi 43: 805-809. 
Tsukaguchi H, Sudhakar A, Le TC, Nguyen T, et al. (2002). NPHS2 mutations in late-onset focal segmental glomerulosclerosis: R229Q is a common disease-associated allele. J. Clin. Invest. 110: 1659-1666.

Wu LQ, Hu JJ, Xue JJ and Liang DS (2011). Two novel NPHS1 mutations in a Chinese family with congenital nephrotic. Genet. Mol. Res. 10: 2517-2522.

Yu ZH, Wang DJ, Meng DC, Huang J, et al. (2012). Mutations in NPHS1 in a Chinese child with congenital nephrotic syndrome. Genet. Mol. Res. 11: 1460-1464. 